\title{
Arsinothricin: a novel arsenic-containing antibiotic
}

\author{
M. Yoshinaga ${ }^{1}$, V.S. Nadar ${ }^{1}$, J. Chen ${ }^{1}$, D.S. Dheeman ${ }^{1}$, B.P. Rosen ${ }^{1}$, \\ M. Kuramata ${ }^{2} \&$ S. Ishikawa ${ }^{2}$ \\ ${ }^{1}$ Herbert Wertheim College of Medicine, Florida International University, Miami, FL, USA \\ ${ }^{2}$ Institute for Agro-Environmental Sciences, NARO, Tsukuba, Ibaraki, Japan
}

\begin{abstract}
The emergence and spread of bacterial resistance highlights the urgent need for new antibiotics. Organoarsenicals have been used as antimicrobials since Paul Ehrlich's salvarsan. Recently a soil bacterium was shown to produce the organoarsenical arsinothricin (AST), a mimetic of the herbicidal antibiotic phosphinothricin (PT). We demonstrate that AST is an effective broad-spectrum antibiotic, showing that bacteria have acquired the ability to utilize environmental arsenic to produce a potent antimicrobial. With every new antibiotic, resistance inevitably arises. The ars $N$ gene, widely distributed in ars operons, confers PT resistance. The functional linkage of $\operatorname{ars} N$ to arsenic detoxification was unclear. Here we show that $\operatorname{ars} N$ selectively confers resistance to AST. Crystal structures of the ArsN $N$-acetyltransferase shed light on the mechanism of selectivity. These results may lead to development of a new class of antimicrobials and ArsN inhibitors.
\end{abstract}

\section{INTRODUCTION}

New antibiotics are urgently needed because the emergence of resistance has rendered nearly every clinically used antibiotic ineffectual. Recently a new arseniccontaining natural product, arsinothricin (AST), was shown to be produced by the rhizosphere microbe Burkholderia sp. GSRB05 (Kuramata et al., 2016). AST is an arsenic mimetic of the Streptomyces phosphonate antibiotic phosphinothricin (PT). The use of arsenicals as antimicrobial and anticancer agents is well-established (Jolliffe, 1993). Here we show that AST is a potent broad-spectrum antibiotic and propose that AST has the potential to be the progenitor of a new class of organoarsenical antibiotics and provide a countermeasure to the return of the pre-antibiotic era.

\section{METHODS}

\subsection{In vivo resistance assays}

Cells were grown in LB medium to log phase, following which the cells were 100-time diluted in M9 medium containing arsenite (As(III)), methylarsenite (MAs(III)), AST or PT. Resistance was determined from the $\mathrm{A}_{600}$ after $24 \mathrm{~h}$ incubation.

\subsection{Gene cloning, protein purification and crystallization and enzyme assays}

Pseudomonas putida KT2440 arsN (PparsN) and the pat gene encoding PT N-acetyltransferase from
Streptomyces viridochromeogenes (Svpat) were cloned into pBAD expression vector and transformed into E. coli TOP10. His-tag PpArsN and SvPAT were purified from the induced cells using a Ni-NTA column chromatography. Crystals of apo- and AST-bound $\mathrm{PpArsN}$ were grown at room temperature. X-ray data were collected on beamline 22ID at the Advanced Photon Source, Argonne National laboratory. The structures were solved by molecular replacement method using PDB ID: 1 YVO as a template. The enzymatic activities and kinetic parameters of the purified PpArsN and SvPAT for PT and AST were determined (Thompson et al., 1987).

\section{RESULTS AND DISCUSSION}

\subsection{AST is a potent broad-spectrum antibiotic}

AST is effective on a variety of Gram-negative and Gram-positive bacterial species at a thirtieth of the concentration of PT, suggesting that AST is a much more effective broad-spectrum antibiotic than PT (Fig. 1). Two bacterial species were resistant to AST. Burkholdeira sp. GSRB05 is the producer of AST, so it is not unreasonable that the species has a mechanism of resistance to AST and PT. As discussed below, the ars $N$ gene confers resistance in $P$. putida KT2440. In E. coli AW3110 $\triangle \operatorname{ars} R B C$, AST was found to be considerably more inhibitory than As(III), and its inhibitory activity was similar to that of highly toxic MAs(III). These results suggest that AST-synthesizing bacteria use environmental inorganic arsenic to produce and secrete AST to kill off competitors. 


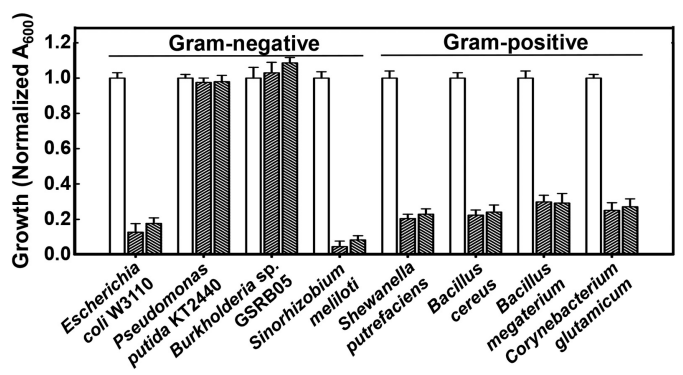

Figure 1. AST is a broad-spectrum antibiotic. Cells were cultured in the absence (left) or presence of $25 \mu \mathrm{M}$ AST (middle) or $800 \mu \mathrm{M}$ PT (right). Data are the mean $\pm \mathrm{SE}$ $(\mathrm{n}=3)$.

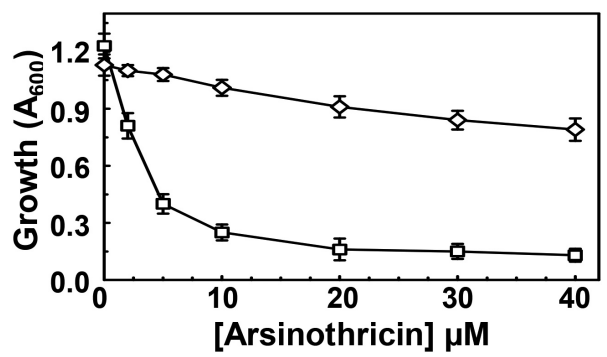

Figure 2. PpArsN confers resistance to AST. Strains: E. coli AW3110 bearing control plasmid $(\square)$ or plasmid carrying the $\operatorname{Ppars} N$ gene $(\diamond)$. Data are the mean $\pm \mathrm{SE}(\mathrm{n}=3)$.

\subsection{ArsN confers AST resistance}

With every antibiotic, resistance inevitably arises. PT resistance is conferred by PT $N$-acetyltransferase (PAT). A gene termed $\operatorname{ars} N$ is found in many arsenic resistance (ars) operons that has been annotated as encoding a PAT. ArsN from P. putida KT2440 (PpArsN) has been demonstrated to inactivate PT by $N$-acetylation (4). Why an enzyme for PT resistance should be in ars operons was a mystery. The identification of AST as a natural product suggested that the biological function of ArsN could be as an AST resistance. We examined the ability of ArsN to confer AST resistance (Fig. 2).

AW3110 is sensitive to AST, whereas AW3110 expressing Ppars $N$ became resistant to AST, supporting our hypothesis that ArsN confers AST resistance. Heterologous expression of PparsN also conferred PT resistance. These results are consistent with the results of P. putida (Páez-Espino et al., 2015) (Fig. 1). In addition, we demonstrated that purified PpArsN is more selective for AST over PT. We compared this with the substrate selectivity of purified PAT from the PTsynthesizing $S$. viridochromeogenes (SvPAT), whose natural substrate is PT. SvPAT showed comparable activity with PT and AST. Thus ArsN, which is selective for AST, is different from PAT, which shows no selectivity.
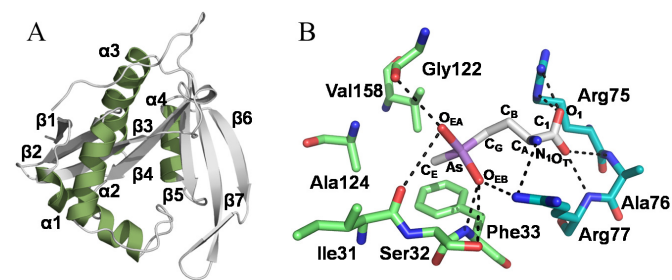

Figure 3. Structure of PpArsN. A: Overall fold of apo-PpArsN. $\alpha$ helices are shown in dark green. B: Interaction of AST with PpArsN. The AST binding site is formed by residues from both the chains A (cyan) and B (green) of the dimer.

\subsection{Crystal structure of ArsN}

To elucidate the mechanism of PpArsN resistance and its selectivity for AST, we solved structures of apo- and AST-bound PpArsN. PpArsN belongs to the GCN5 $N$-acetyltransferase (GNAT) superfamily and like other GNAT superfamily members, the overall conformation of PpArsN is an $\alpha / \beta$ three-layer sandwich fold (Fig. 3A). PpArsN forms an asymmetric homodimer, as does PAT. Although PpArsN shares low protein sequence identity $(\sim 35 \%)$ with PAT, the backbone structures of these proteins are well conserved. PpArsN has two AST-binding sites, which are asymmetrically formed by amino acid residues from both Chains A and B. Both active sites are composed of nine residues: six residues from one chain and three residues from the other (Fig. 3B). Comparison of the structures of AST-bound PpArsN and PTand CoA-bound PAT from S. hygroscopicus (ShPAT) (PDB ID: 5T7E) (Christ et al., 2017) demonstrates similarities of the active sites of these two related $N$-ace-tyltransferases.

The residues involved in AST binding in PpArsN are conserved well in ShPAT, with several conservative replacements. Surprisingly, sets of amino acid residues used by ShPAT to interact with each chemical moiety in PT are different from those used by PpArsN to interact with the corresponding chemical moiety in AST. As a result, while the arsenic atom of AST overlaps with the phosphorus atom of PT, the orientation of the amino group of $\mathrm{PT}$ is inclined to $95^{\circ}$ towards the acetyl-CoA binding site with respect to the AST binding site. The minor difference in composition of amino acid residues forming the substrate binding site between ArsN and PAT may lead in part to the variation of their substrate selectivity.

\section{CONCLUSIONS}

Our results demonstrate that the novel organoarsenical natural product AST is a broad-spectrum antibiotic much more potent than its phosphorus mimetic PT. We also show that ArsN selectively confers resistance to AST over PT, elucidating the functional linkage of ArsN to arsenic detoxification. Our ArsN crystal 
structures will be useful to design new drugs that can evade resistance or enhance AST effectiveness as antibiotics.

\section{ACKNOWLEDGEMENTS}

This work was supported by NIH grant R01 GM055425 to BPR, Herbert Wertheim College of Medicine Pilot Fund (\#800008403) to MY, and a JSPS KAKENHI grant (No. 23380044) to SI.

\section{REFERENCES}

Christ, B., Hochstrasser, R., Guyer, L., Francisco, R., Aubry, S., Hörtensteiner, S. \& Weng, J.-K. 2017. Non-specific activities of the major herbicide-resistance gene BAR. Nat. Plants 3(12): 937.
Jolliffe, D.M. 1993. A history of the use of arsenicals in man. J. Roy. Soc. Med. 86(5): 287-289.

Kuramata, M., Sakakibara, F., Kataoka, R., Yamazaki, K., Baba, K., Ishizaka, M., Hiradate, S., Kamo, T. \& Ishikawa, S. 2016. Arsinothricin, a novel organoarsenic species produced by a rice rhizosphere bacterium. Environ. Chem. 13(4): 723-731.

Páez-Espino, A.D., Chavarría, M. \& Lorenzo, V. 2015. The two paralogue phoN (phosphinothricin acetyl transferase) genes of Pseudomonas putida encode functionally different proteins. Environ. Microbiol. 17(9): 3330-3340.

Thompson, C.J., Movva, N.R., Tizard, R., Crameri, R., Davies, J.E., Lauwereys, M. \& Botterman, J. 1987. Characterization of the herbicide-resistance gene bar from Streptomyces hygroscopicus. EMBO J. 6(9): 2519-2523. 